\title{
The Alert Stethoscope
}

\author{
Ria Kaur Ajmani, \\ Student, ECE branch, \\ Kakatiya institute of technological sciences. \\ Telangana ,India.
}

\begin{abstract}
The main idea of this alert stethoscope is that during this pandemic, effected by the deadly virus has got the most of patients and the whole of doctors into the emergency needs of this virus. But there's an equal rate of other general diseases and patients who cannot be ignored surely, this is a chaotic situation for the doctors but can make it little easier for the doctors to know their emergencies simply through their stethoscope. Without any structural modifications this system can be embedded inside the stethoscope. This can also be applicable in many fields like Using digital jewelry and Alert systems at other public places etc.
\end{abstract}

Keywords:- AC Oscillator, Alert codes, Antenna, Buzzer, Capacitor, Crystal Oscillator, Digital Jewelry, Incandescent Lamp, Inductor, LCD Display, Memory Buzzer, OCS Timer, Paging Signal Receiver, Paging Signal Transmitter, Processor, Renne Laennec, Resistor, Switch Buzzer, SPST Push Button, Transformer, TransistorBipolar NPN

\section{INTRODUCTION}

The usage of gadgets has been growing enormously in today's world. When you compare the size of today's electronic devices with that of ten years back, you can notice the advancements that took place in the world of Technology and Medical services. We all knew that Stethoscope is a symbol of healthcare professionals. This new "Alert Stethoscope" is designed to supplement the needs of patient's emergencies according to the availability of doctors. And can also include hospital emergencies for the beneficiary of the doctors and patients. There wouldn't be any way easier and earlier to receive an emergency alert for the doctors.

\section{RELATED TECHNOLOGIES AND ARCHITECTURES}

\section{$>$ Digital Jewelry}

Digital jewelry is the fashion jewelry with embedded intelligence. It can best be defined as wireless, wearable computers that allow you to communicate by ways of email, voicemail, and voice communication. [4] functions:

The pieces of computerized-jewelry phone and their

- Earrings - Speakers embedded into these earrings will be the phone's receiver.

- Necklace - Users will talk into the necklace's embedded microphone.

\author{
Manda Rishita, \\ Student, CSE \\ Kakatiya institute of technological sciences. \\ Telangana, India.
}

- Ring - Perhaps the most interesting piece of the phone, this "magic decoder ring, is equipped with lightemitting diodes (LEDs) that flash to indicate an incoming call. It can also be programmed to flash different colors to identify a particular caller or indicate the importance of call.

- Bracelet - Equipped with a video graphics array (VGA) display, this wrist display could also be used as a caller identifier that flashes the name and phone number of the caller.

\section{PROPOSED MODEL AND IMPLEMENTATION DETAILS}

\section{Circuit Components Used}

AC oscillator, source, Antenna, external, Buzzer, Capacitor, Crystal Oscillator, Inductor, Air core, Lamp, incandescent, OCS Timer, Resistor SPST, Push button, Switch, Transformer, Iron core, Transistor, Bipolar, NPN

\section{Function Components}

- Antenna: The Antenna captures some of the RF radiation transmitted by the paging transmitter and feeds it to the receiver.

- Receiver: The receiver removes the information from the Rf carrier (demodulates it) and sends the encoded information to the processor.

- Processor: The processor is "THE BRAIN" of the pager. It decodes the information from the receiver and reproduces the original information sent from the paging transmitter. It checks to see if the message is intended for it and if it is then it transmits it.

- Memory: It stores the message in the electronic memory. It can store many messages depending on memory size. For example it typically stores 50 messages.

- Display: The stored message is then usually displayed on a LCD (liquid crystal display). The processor will display the most recent message first, or some display the most emergency message first.

- Indicators: There are two ways to indicate that a new message has been received.

$\checkmark$ Audible beeper

$\checkmark$ Inaudible vibrator

$\checkmark$ The vibrator notifies you silently, when the beeping noise would be intrusive. You can configure these indicators to be on or off or an easily recognizable pattern that tells you how urgent the message is, without you having it to look at the display. 


\section{Method of Actions/The procedure}

Emergency (patient /ambulance): The need of emergency is generally informed to the hospital authorities.

Hospital Administration: It uses an antenna which is a high frequency transmitter base which sends stability frequency with DSP (digital signal processing) which is 250 watts $/ 900 \mathrm{MHz}$ or $1100 \mathrm{MHz}$. It will then send the message to certain Doctor with the help of Doctor Availability register. Each Doctor has a specific code which is only specific to him.

- The radio frequency is set from $900-1000$ which is localized radio frequency.

- The message of emergency is then sent by using Paging signal Controller [3]. Then it is transmitted further by paging signal transmitter.

Doctor alert receiver: Doctor gets an alert message in the form of numbers. Ex: 9999 and, so the alert is received by the doctor. This is less time consuming and more beneficial to the doctors.

The alert codes are segregated based on the specifications of doctors for the emergency of the patients. There are other hospital general emergencies which can be improvised using these codes.

The general hospital codes for emergencies are

- Code pink (reads 9999): infant or child abduction

- Code orange (reads 8888): Hazardous material or spill accident

- Code silver (reads 7777): Active shooter.

- Code Violet (reads 6666): violent or combative individual.

- Code yellow (reads 5555): Disaster

- Code brown (reads 4444): incoming patient (emergency).

- Code white (reads 3333): evacuation

- Code green (reads 2222): emergency activation.

\section{Circuit Diagram}

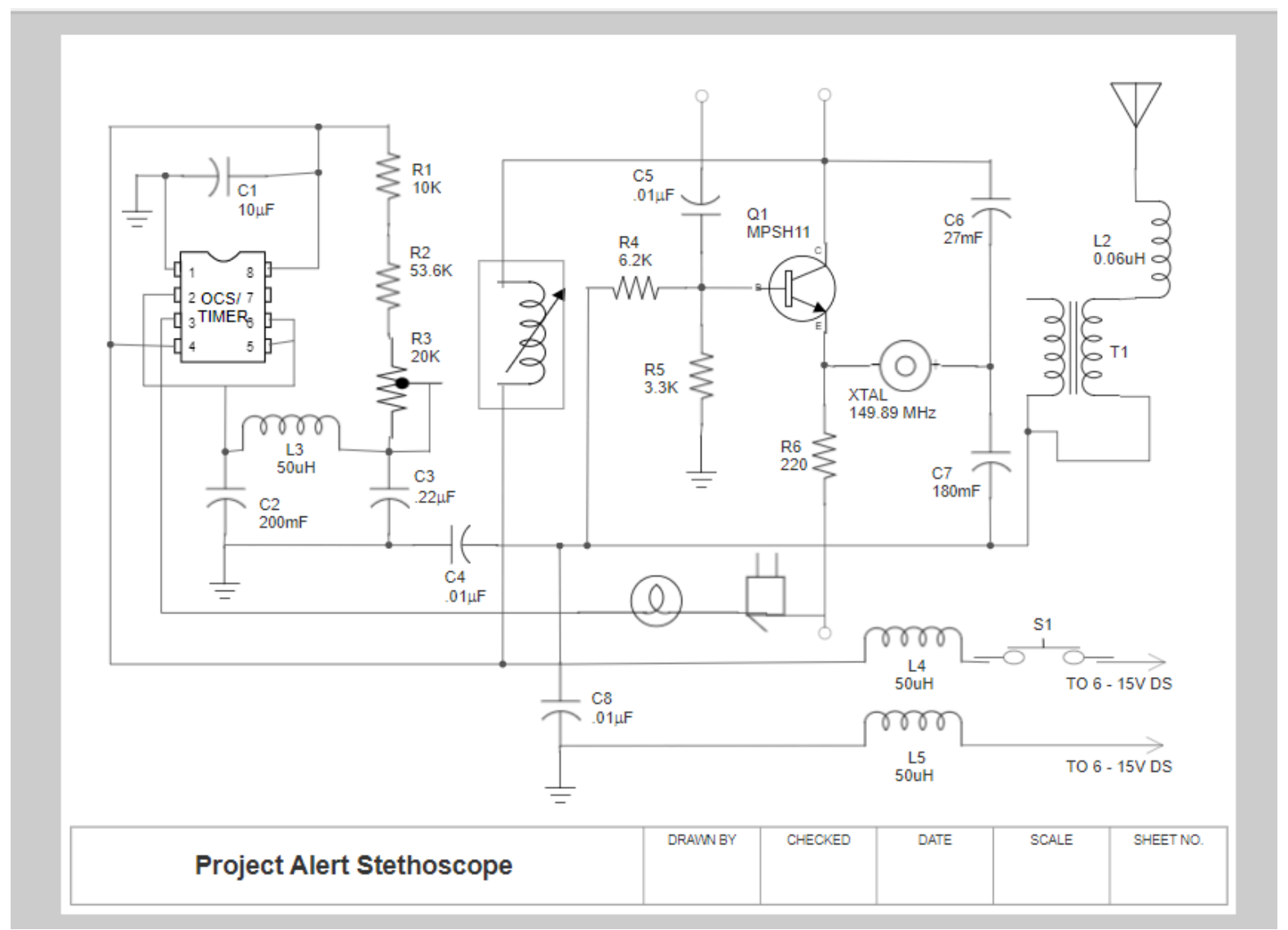

Fig 1 


\section{Device Outlook}

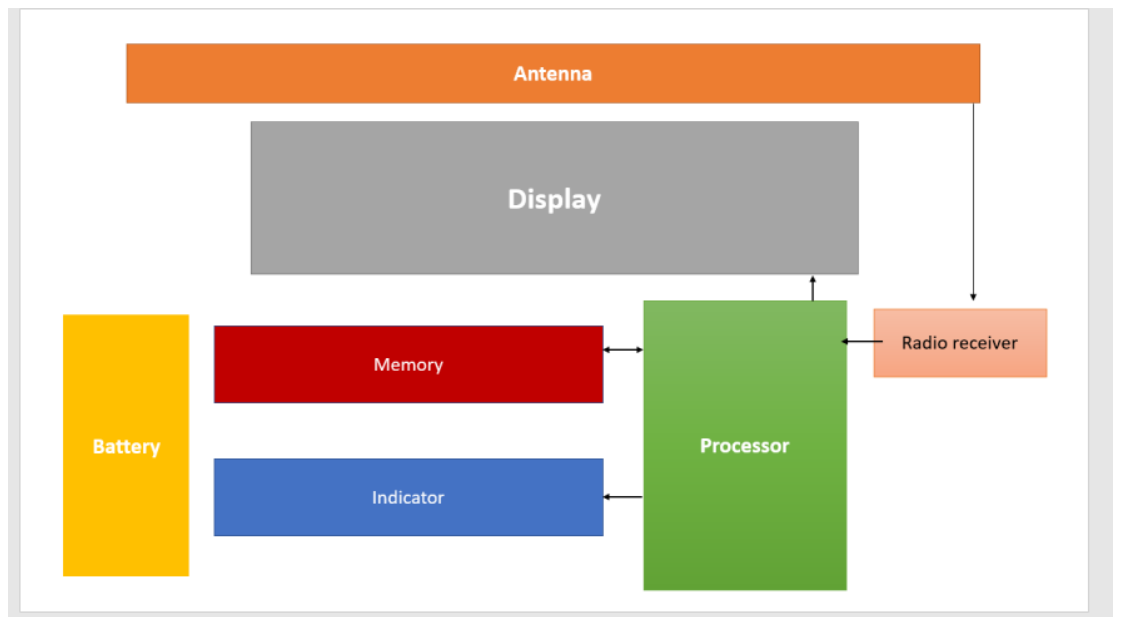

Fig 2

\section{Method of Action}

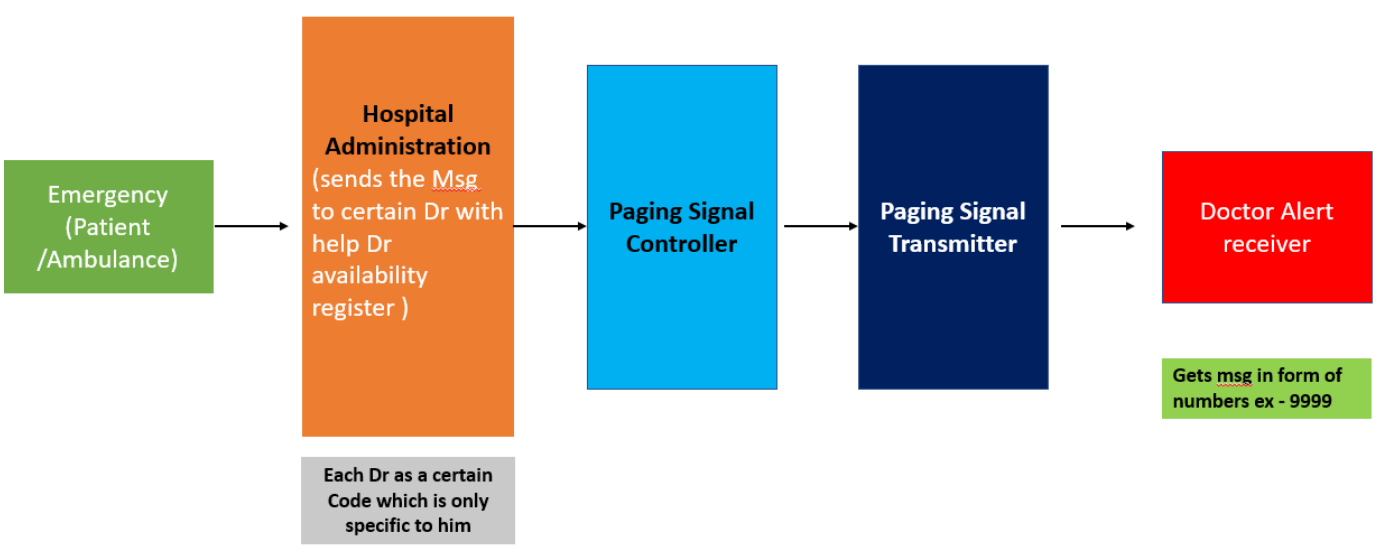

Fig 3

\section{Embedding of this Device}

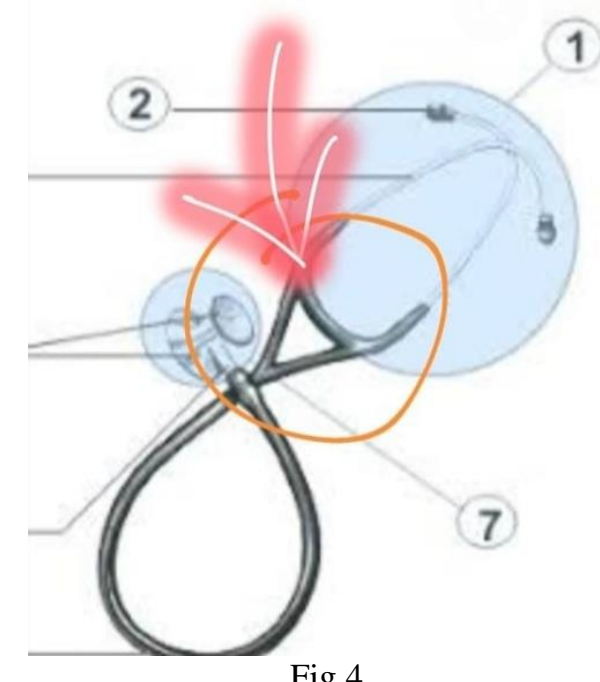

Fig 4 
RED ARROW: It can be embedded in another way by adding a triangular device in between $\mathrm{V}$ and $\mathrm{U}$ bifurcation.

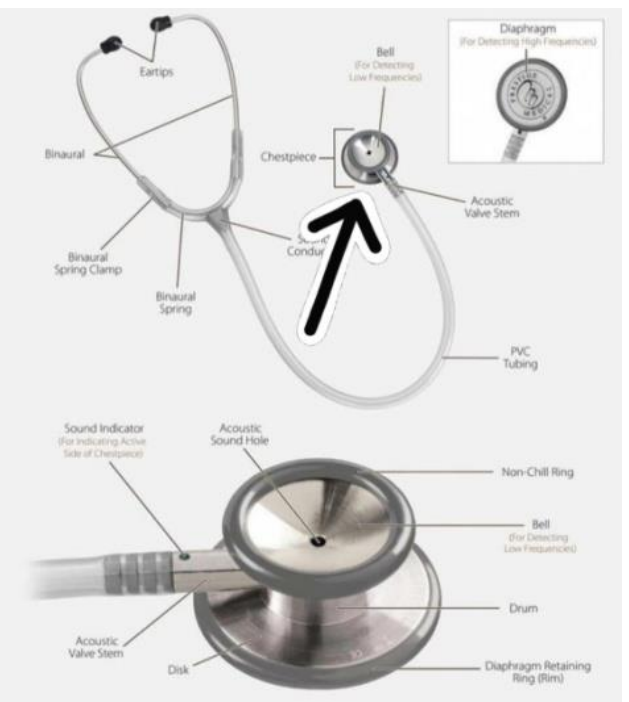

Fig 5

BLACK ARROW: It can be embedded in the diaphragm

\section{STATISTICAL TESTS AND PARAMETERS}

There must be a legal permission from the WHO to make any changes or implement any new technology into the equipments. The work must be ethical prior to any other aspects. The circuit, its connections and the parameters of setting it up must be done vigilantly.

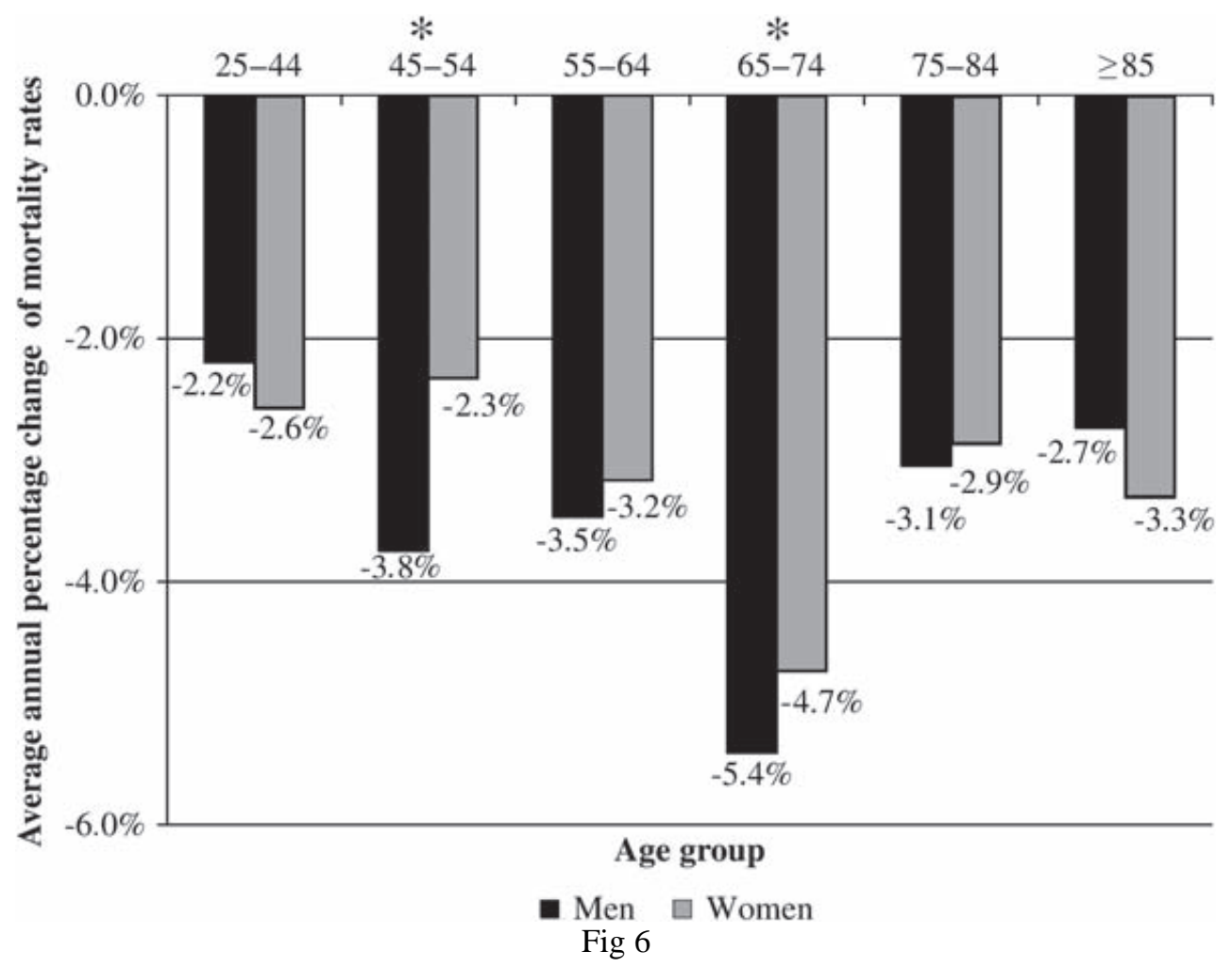

The above graph [5] is an example of the Average annual percentage change of mortality rates due to heart failure in both men and women of different age groups. Even in a pandemic of this deadly virus, this is an example of the emergency of other non virus affected patients. This is chaotic situation for doctors to attend all the emergencies. So a simple technological improvisation of an emergency alert using stethoscope as the alert receiver could be more effective. 


\section{RESULTS AND DISCUSSIONS}

An Alert stethoscope can be made by embedding few digital devices, without any changes in the model or functioning of the stethoscope. The easily and less time consuming emergency alert to a doctor using stethoscope could be the best and beneficial way to the doctors. The device can be embedded in diaphragm without any disturbances in PVC tubing. It can also be embedded in another way. We can add a triangular device in between $\mathrm{V}$ and $U$ bifurcation. The emergencies can be related to the patient's emergencies or the hospital emergencies. This can also be done by embedding an alarm buzzer inside the stethoscope. Further modifications or new embedding of devices can be done depends on one's choice.

The circuit can be embedded in the Diaphragm or else in another way by adding a triangular device between $\mathrm{V}$ and $\mathrm{U}$ Bifurcation.

\section{CONCLUSION}

Doctors will not have to struggle with being up- todate in medical research and with administrative tasks. It takes a while for the medical community to accept the stethoscope. It has the potential to revolutionize health care; it will displace the stethoscope as its symbol.

\section{REFERENCES}

[1]. https://www.fema.gov/emergency-alert-system

[2]. https://en.wikipedia.org/wiki/Stethoscope

[3]. http://braddye.com/pagsys/pagsys.html\#: : text=The\% 20paging\%20base $\% 20$ stations $\% 20$ decode,transmissio n\%20to\%20the\%20paging\%20receiv

[4]. https://krazytech.com/technical-papers/digital-jewelry

[5]. https://onlinelibrary.wiley.com/doi/pdf/10.1002/ejhf.2 84\#: :text=The $\% 20$ overall $\% 20$ crude $\% 20$ rate $\% 20$ of,th an\%20in\%20women\%20(316.2\%20vs.

[6]. https://www.researchgate.net/publication/275208316_ 270_MI 